\title{
Regulation of mammalian MOR-1 gene expression after chronic treatment with morphine
}

\author{
ROSE V. PRENUS, EBENS LUSCAR, ZHI-PING ZHU, RAMESH B. BADISA and CARL B. GOODMAN \\ College of Pharmacy and Pharmaceutical Sciences, Florida A\&M University, Tallahassee, FL 32307, USA
}

Received April 9, 2012; Accepted June 5, 2012

DOI: $10.3892 /$ ijmm.2012.1132

\begin{abstract}
Morphine is an effective analgesic that acts by binding to the $\mu$-opioid receptor (MOR) coded in the human by the OPRM1 gene. In the present study, we investigated the regulation of $\mu$-opioid receptor (MOR-1) mRNA levels in all-trans-retinoic acid-differentiated SH-SY5Y human neuroblastoma cells under in vitro conditions with $10 \mu \mathrm{M}$ morphine treatment for $24 \mathrm{~h}$. In addition, we measured the MOR-1 levels in recombinant Chinese hamster ovary (CHO) cells, transfected with human $\mu$-opioid receptor gene (hMOR) with $10 \mu \mathrm{M}$ morphine treatment for $24 \mathrm{~h}$. The isolated mRNA from these cells was subjected to real-time quantitative RT-PCR analysis to determine the regulation of $\mu$-opioid receptor gene expression. It was observed that morphine treatment did not alter MOR-1 levels in undifferentiated SH-SY5Y cells compared to undifferentiated control cells. However, the MOR-1 levels in all-trans-retinoic acid-differentiated cells were significantly higher compared to the undifferentiated cells. Morphine treatment in differentiated SH-SY5Y cells caused significant downregulation of MOR-1 expression compared to the control cells. In the morphine-treated CHO cells, the hMOR-1 mRNA levels remained the same as the untreated control. Finally, pretreatment of SH-SY5Y cells with $10 \mu \mathrm{M}$ naloxone, the antagonist of $\mu$-opioid receptor, for $1 \mathrm{~h}$ significantly blocked the downregulation of MOR-1 mRNA levels with morphine treatment. These findings suggest that regulation of MOR-1 gene expression is cell-type specific after chronic morphine treatment and provide some evidence in the understanding of morphine tolerance.
\end{abstract}

Correspondence to: Dr Carl B. Goodman, College of Pharmacy and Pharmaceutical Sciences, Florida A\&M University, Tallahassee, FL 32307, USA

E-mail: carl.goodman@famu.edu

Abbreviations: CNS, central nervous system; FBS, fetal bovine serum; MOR-1, $\mu$-opioid receptor; PBS, phosphate-buffered saline; RA, all-trans-retinoic acid; WHO, World Health Organization

Key words: morphine, naloxone, $\mu$-opioid receptor, real-time qRT-PCR

\section{Introduction}

Opium, the substance derived from the poppy plant, Papaver somniferum, has been used in medicine as an analgesic agent of pain reliever in different parts of the world for over 6000 years. Morphine is one of the highly potent and abundant alkaloids present in the opium, and is responsible for analgesic property. As early as eighteenth century, morphine was used in surgical procedures and pain management. The World Health Organization (WHO) recommended its use for controlling pain in cancer patients (1). Since morphine also produces euphoric feeling, it has become one of the highly abused drugs in the world currently. Long-term or chronic use of morphine is shown to associate with drug tolerance (2). Drug tolerance not only limits the use of morphine in clinical application but also involves tragic circumstances in drug addicts. In vivo studies indicated that morphine alters gene transcription in the brain (3) and spinal cord after acute and chronic administration. Previous studies have demonstrated that morphine induces long-term changes in neurons (4).

It is widely believed that the behavioral changes in drug addicts could be due to the altered gene expression in central nervous system (CNS). Studies demonstrated that $\mu$-opioid receptor (MOR-1) is the primary site of action for morphine and the other most commonly used opioids $(5,6)$. The process of morphine tolerance is very complex (7), but from the clinical point of view, it is important to understand the mechanism of its tolerance, because it may lead to treatment and prevention of opiate addiction. The MOR-1 gene expression is regulated at the level of DNA transcription or post-transcription. Since the short-term morphine treatment does not downregulate the MOR-1 receptor (8), in the present work, we studied the long-term chronic morphine treatment for drug tolerance mechanism on the regulation of MOR-1 in SH-SY5Y cells and $\mathrm{CHO}$ cells at the post-transcriptional level. In addition, we also investigated the effect of morphine on the regulation of MOR-1 receptor mRNA levels in the presence of receptor antagonist naloxone.

\section{Materials and methods}

Materials. Morphine sulfate, naloxone hydrochloride and all-trans-retinoic acid were obtained from Sigma-Aldrich ${ }^{\circledR}$ (St. Louis, MO, USA). All other routine chemicals and reagents used were of analytical grade. 
Cell cultures. The human neuroblastoma cells (SH-SY5Y) were purchased from the American Type Culture Collection (Manassas, VA, USA). The recombinant Chinese hamster ovary (CHO) cells, transfected with human $\mu$-opioid receptor gene (hMOR), were a kind gift from Dr Richard Rothman, NIDA-NIH Addiction Research Center (Baltimore, MD, USA). Both cell-types were maintained separately as adherent monolayer cultures. The SH-SY5Y cells were grown in the media without phenol-red, in a ratio of 1:1 mixture of Dulbecco's modified Eagle's medium (DMEM) and Ham's F12 medium (Invitrogen, Molecular Probes, Eugene, OR, USA), with $2.5 \mathrm{mM}$ L-glutamine, $0.5 \mathrm{mM}$ sodium pyruvate, and $1200 \mathrm{mg} / \mathrm{l}$ sodium bicarbonate, supplemented with $10 \%$ FBS, penicillin $(100 \mu \mathrm{g} / \mathrm{ml})$ and streptomycin $(100 \mathrm{U} / \mathrm{ml})$. The recombinant $\mathrm{CHO}$ cells, transfected with hMOR-1 gene, were grown in the same media in a ratio of 1:1 as described above, containing phenol-red. The medium was supplemented with $10 \%$ FBS, penicillin $(100 \mu \mathrm{g} / \mathrm{ml})$ and streptomycin (200-250 U/ml). During experimental studies with CHO cells, the phenol-red free medium was employed, supplemented with all components as mentioned above. The cultures were maintained in an atmosphere of humidified air with $5 \% \mathrm{CO}_{2}$ at $37^{\circ} \mathrm{C}$ in an incubator.

Differentiation of SH-SY5Y cells. The neuroblastoma cells $\left(5 \times 10^{5}\right)$ were seeded in culture dishes in complete medium $(30 \mathrm{ml})$, and allowed to grow until the cells reached $70-80 \%$ confluence. All-trans-retinoic acid (RA) was dissolved in $95 \%$ ethanol as a stock of $10 \mathrm{mM}$. A known volume of RA stock was added to the cultures to attain a final concentration of $10 \mu \mathrm{M}$ (9). Control cells received an equal volume of the vehicle $(0.1 \%)$. All culture dishes were incubated for $72 \mathrm{~h}$ continuously without further renewal of growth medium in the incubator.

Treatments with morphine in SH-SY5Y cells. Morphine sulfate was dissolved in deionized water as a $10 \mathrm{mM}$ stock and added to the cultures to achieve a final concentration of $10 \mu \mathrm{M}$ (10). Control cells received an equal volume of the vehicle. In some of the experiments, the cells were pre-treated with $10 \mu \mathrm{M}$ naloxone hydrochloride (MOR-antagonist) for $1 \mathrm{~h}$, followed by treatment with $10 \mu \mathrm{M}$ morphine sulfate for $24 \mathrm{~h}$.

Treatments with morphine in recombinant $\mathrm{CHO}$ cells. The CHO cells were seeded in culture dishes in complete medium devoid of phenol-red. To the cells, a known volume of morphine stock was added to the cultures to attain a final concentration of $10 \mu \mathrm{M}(10)$. Control cells received an equal volume of the vehicle. All culture dishes were incubated for $24 \mathrm{~h}$ continuously without further renewal of growth medium in the incubator.

RNA isolation. At the end of $24 \mathrm{~h}$ treatment, cells were washed three times with PBS to remove the drug compounds and the serum proteins. Then the cells were harvested using cell scrapers, and centrifuged at $1500 \mathrm{rpm}$ for $3 \mathrm{~min}$. The cell-pellets were re-suspended in $1 \mathrm{ml}$ PBS, and transferred into eppendorf tubes, and subjected to centrifugation at $1000 \mathrm{rpm}$ for $3 \mathrm{~min}$. Finally, the pellets were homogenized in $1 \mathrm{ml}$ TRIzol reagent with VirTishear polytron homogenizer
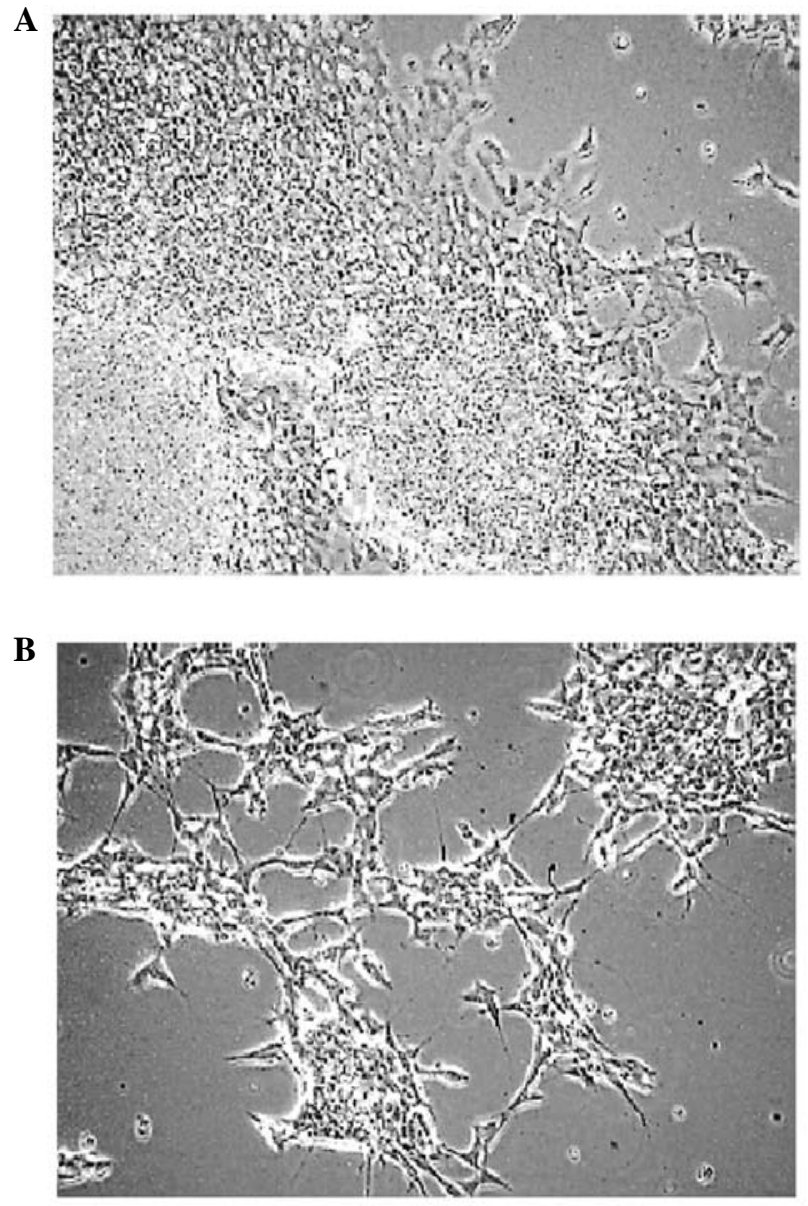

Figure 1. Morphology of undifferentiated and differentiated human SH-SY5Y cells. Cells were grown in culture dishes in the complete medium without RA (A) or with $10 \mu \mathrm{M}$ RA (B). Unstained cells were photographed after $72 \mathrm{~h}$ of RA treatment using an inverted phase contrast IX-70 Olympus microscope. Neurite extensions in RA-induced differentiation (B) were seen clearly.

Table I. Sequence of the primers used in real-time PCR for human SH-SY5Y cells.

\begin{tabular}{ll}
\hline mRNA & \multicolumn{1}{c}{ Primers } \\
\hline MOR-1 & F: 5'-ATGCCAGTGCTCATCATTAC-3' \\
& R: 5'-GATCCTTCGAAGATTCCTGTCCT-3' \\
$\beta$-actin & F: 5'-GATGAGATTGGCATGGCTTT-3' \\
& R: 5'-CACCTTCACCGTTCCAGTTT-3' \\
\hline
\end{tabular}

(Virtis Company, Inc., Cardiner, NY, USA). Total RNA was extracted with chloroform and isopropanol according to the manufacturer's instructions (Invitrogen, Carlsbad, CA, USA). Following ethanol precipitation, the vacuum-dried RNA was dissolved in $100 \mu \mathrm{l}$ of DEPC-water. The quantity of total RNA was measured by the Nanodrop ND-1000 spectrophotometer (NanoDrop Technologies, Wilmington, DE, USA). RNA was subjected to DNAase treatment for $30 \mathrm{~min}$ at $37^{\circ} \mathrm{C}$ using DNase Treatment and Removal Reagent (Ambion, Austin, TX, USA). The purified RNA with A260/A280 ratio of $\geq 1.8$ was subsequently used for cDNA synthesis. 


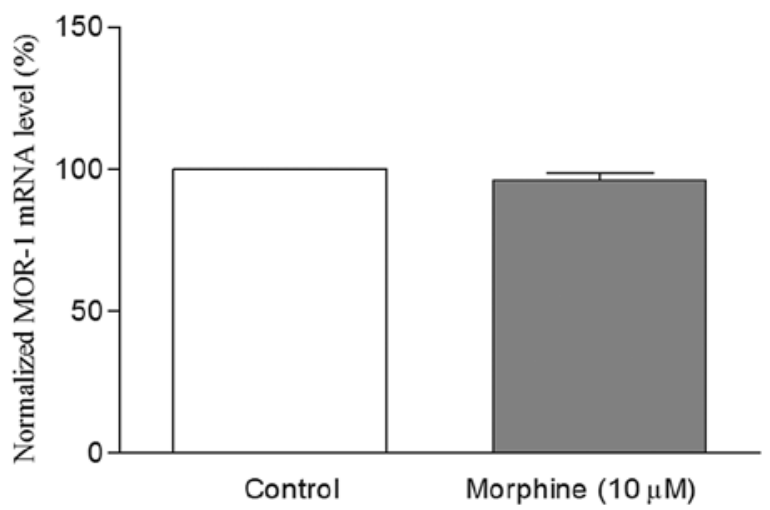

Figure 2. Effect of morphine on MOR-1 mRNA levels in undifferentiated SH-SY5Y cells. Cells were treated with $10 \mu \mathrm{M}$ morphine for $24 \mathrm{~h}$ in the complete medium. MOR-1 relative mRNA levels were quantified by real-time $\mathrm{PCR}$ using $\beta$-actin as a reference gene. Data are presented as the mean \pm SEM $\left(\mathrm{n}=3,{ }^{*} \mathrm{P}>0.05\right.$, insignificant in comparison to control, one-way ANOVA, Student's t-test.

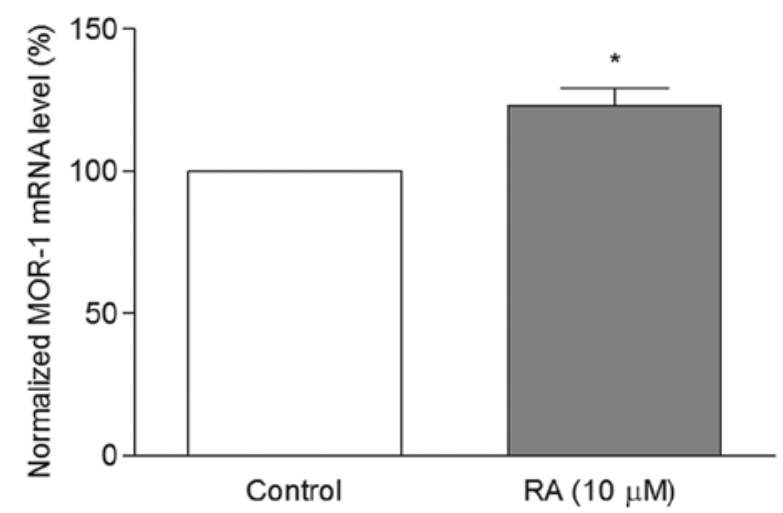

Figure 3. MOR-1 mRNA levels in undifferentiated (control) and differentiated SH-SY5Y cells. Cells were differentiated with $10 \mu \mathrm{M}$ RA for $72 \mathrm{~h}$ MOR-1 relative mRNA levels were quantified by real-time PCR using $\beta$-actin as a reference gene. Data are presented as the mean \pm SEM $\left(n=3,{ }^{*} \mathrm{P}<0.05\right.$, significant in comparison to control, one-way ANOVA, Student's t-test.

cDNA synthesis. The cDNA synthesis was performed with an iScript cDNA synthesis kit (Bio-Rad, Hercules, CA, USA) using $10 \mu \mathrm{g}$ of total RNA according to the manufacturer's instructions.

Data analyses. Data were presented as mean \pm standard error of the mean (SEM). Differences between the means were compared by Student's t-test. Statistical significance is ascribed for $\mathrm{P}<0.05$. Curve fitting was conducted using GraphPad Prism 3.02 (GraphPad Software Inc., San Diego, CA).

\section{Results}

Morphological differentiation of SH-SY5Y cells. It was observed that the undifferentiated neuroblastoma cells grew mostly together in the form of clumps (Fig. 1A). On the other hand, cells treated with all-trans-retinoic acid (RA) for $72 \mathrm{~h}$ resulted in a significant cellular differentiation, characterized with elongated neurites (Fig. 1B). The concentration of RA was based on previous studies (10). Our results clearly demonstrate that RA promotes SH-SY5Y cell differentiation.

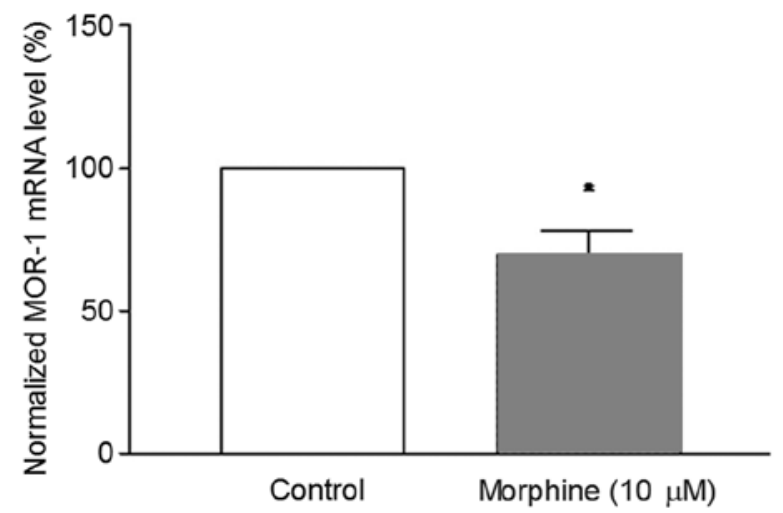

Figure 4. Effect of morphine on MOR-1 mRNA levels in differentiated SH-SY5Y cells. Cells were differentiated with $10 \mu \mathrm{M}$ RA for $72 \mathrm{~h}$, followed by treatment with $10 \mu \mathrm{M}$ morphine for $24 \mathrm{~h}$ in the complete medium. MOR-1 relative mRNA levels were quantified by real-time PCR using $\beta$-actin as a reference gene. Data are presented as the mean $\pm \operatorname{SEM}\left(n=3,{ }^{*} P<0.05\right.$, significant in comparison to control, one-way ANOVA, Student's t-test.

Regulation of MOR-1 gene expression in SH-SY5Y cells. The mRNA levels of MOR-1 were quantitated by real-time RT-PCR using MOR-1 specific primers listed in Table I. $\beta$-actin, a housekeeping gene, was used for the normalization of gene expression. It was observed that the MOR-1 mRNA levels in undifferentiated cells with $10 \mu \mathrm{M}$ morphine treatment for $24 \mathrm{~h}$ remained the same as those of the undifferentiated control cells (Fig. 2). However, in the RA differentiated cells, these levels were significantly increased in comparison to the undifferentiated control cells (Fig. 3). These results clearly show that the MOR-1 mRNA levels depend on the cellular differentiation. When the differentiated cells were treated with $10 \mu \mathrm{M}$ morphine for $24 \mathrm{~h}$, the MOR-1 mRNA levels were significantly reduced compared to differentiated control cells (Fig. 4). The results indicate that the MOR-1 gene regulation with morphine treatment depends on the cellular differentiation.

Regulation of MOR-1 gene expression in hMOR-CHO cloned cells. In an effort to understand the extent of MOR-1 gene regulation in a different cell system, we used Chinese hamster ovary (CHO) cells that were transfected with hMOR which stably express the MOR protein. The cells were treated with $10 \mu \mathrm{M}$ morphine for $24 \mathrm{~h}$, and the mRNA levels were measured by real-time RT-PCR. It was found that morphine treatment caused decrease in the mRNA levels (Fig. 5). However, this decrease was not significant $(\mathrm{P}>0.05)$. Therefore, the $\mathrm{CHO}$ cells were not utilized in our further studies.

Reversal of MOR-1 mRNA downregulation by naloxone. The effect of naloxone, which is an antagonist for MOR, was studied on the regulation of MOR-1 gene expression in morphinetreated cells. For this purpose, the differentiated human SH-SY5Y cells were pretreated with $10 \mu \mathrm{M}$ naloxone for $1 \mathrm{~h}$, followed by co-treatment with $10 \mu \mathrm{M}$ morphine for $24 \mathrm{~h}$. It was observed that morphine treatment caused a significant decrease in the MOR-1 mRNA levels, while naloxone alone did not alter these levels. However, in naloxone pretreated cells, morphine treatment did not decrease the MOR-1 mRNA levels (Fig. 6), and remained almost the same levels as those of the control. 


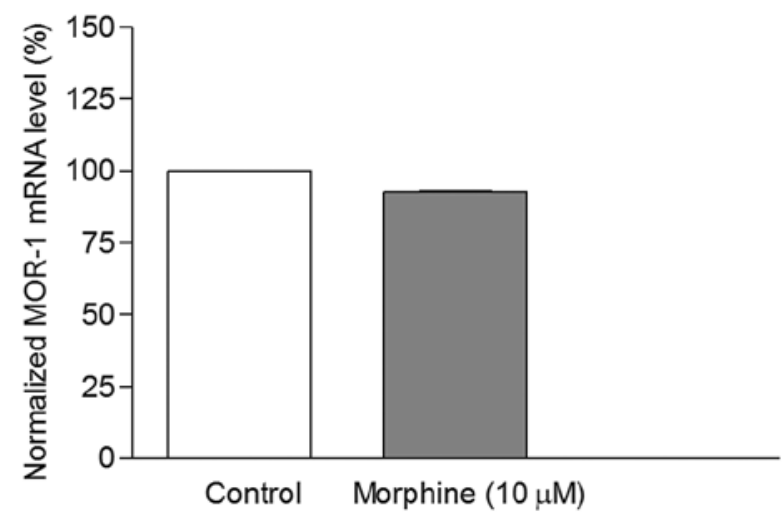

Figure 5. Regulation of MOR-1 mRNA levels in hMOR-CHO cells. Cells were treated with $10 \mu \mathrm{M}$ of morphine for $24 \mathrm{~h}$. MOR-1 relative mRNA levels were quantified by real-time PCR using $\beta$-actin as a reference gene. Data are presented as the mean \pm SEM $\left(n=3,{ }^{*} P>0.05\right.$, insignificant in comparison to control, one-way ANOVA, Student's t-test.

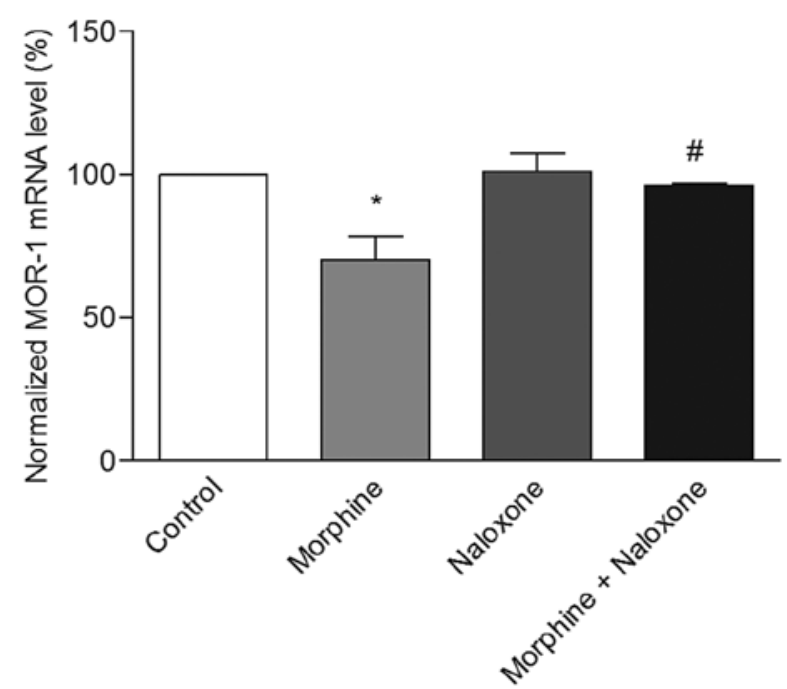

Figure 6. Reversal of MOR-1 mRNA downregulation by naloxone. SH-SY5Y cells were differentiated with $10 \mu \mathrm{M}$ RA for $72 \mathrm{~h}$ in the complete medium, followed by pre-treatment with $10 \mu \mathrm{M}$ naloxone for $1 \mathrm{~h}$. Thereafter, these cells were treated with $10 \mu \mathrm{M}$ morphine for $24 \mathrm{~h}$. MOR-1 relative mRNA levels were quantified by real-time PCR using $\beta$-actin as a reference gene. Data are presented as the mean \pm SEM $(n=3, P<0.05$ : *Significant in comparison to untreated control; "Significant in comparison morphine treated cells, one-way ANOVA, Student's t-test.

These results clearly show that naloxone reverses the morphineinduced downregulation of MOR-1 gene expression.

\section{Discussion}

In the present study, the SH-SY5Y neuroblastoma cells were used as an in vitro model to investigate the effect of morphine on MOR-1 gene expression. This cell line was originally derived from neuroblastoma SK-N-SH clone, and expresses $\mu$ and $\delta$ receptors (11). Usually, the SH-SY5Y cells are differentiated by all-trans-retinoic acid (RA) to achieve neurite-outgrowth and morphological features (12). Both differentiated and undifferentiated SH-SY5Y cells were used as model cultures in neuroscience research (13-16). This posed a selection problem between differentiated and undifferentiated SH-SY5Y cells for our studies.

In order to find a suitable answer for this question, we first compared the MOR-1 gene expression levels in undifferentiated SH-SY5Y cells with morphine treatment. It was observed that morphine treatment did not alter the MOR-1 mRNA levels in these cells compared to undifferentiated control cells (Fig. 2). However, the levels were significantly upregulated in RA differentiated SH-SY5Y cells compared to the undifferentiated cells (Fig. 3). The results highlight that the process of differentiation appears to modulate the response to morphine treatment. These observations were consistent with previous report, where MOR-1 mRNA levels were shown to upregulate in RA differentiated SH-SY5Y cells (17). Since the MOR-1 levels were higher in the differentiated cells than the undifferentiated cells, we preferred to differentiate the cells with RA for further studies.

The morphological features of differentiated cells clearly showed that the cells have elongated neurite extensions (Fig. 1), which are in agreement with previous reports $(18,19)$. We next studied the effect of morphine on MOR-1 mRNA levels in the differentiated cells. It was found that morphine downregulated the MOR-1 levels significantly (Fig. 4). The downregulation of MOR-1 with morphine treatment was also observed earlier in different cell lines (20-22).

We further studied the effect of morphine in recombinant CHO cells for MOR-1 mRNA levels. Morphine treatment did not alter the mRNA levels significantly in these cells (Fig. 5). The results clearly suggest that regulation of MOR-1 gene expression is cell-type specific. Earlier studies on recombinant $\mathrm{CHO}$ cells confirmed our results in terms of having no alteration in mu opoid receptor protein with morphine treatment (23).

Since morphine treatment caused downregulation of MOR-1 mRNA levels in our study, we investigated the compounds that act as antagonist to MOR-1 receptor to prevent the downregulation of MOR-1 gene. Naloxone, an opioid antagonist, was employed in our studies with $1 \mathrm{~h}$ pretreatment, prior to morphine treatment. It was observed that naloxone-pretreatment blocked the downregulation of MOR-1 gene expression significantly (Fig. 6). Similar observation was reported earlier, where naloxone was shown to block the downregulation of receptor protein with morphine treatment (10).

In conclusion, chronic morphine treatment caused the downregulation of MOR-1 gene expression in human differentiated SH-SY5Y cells, while naloxone reversed this process. The results clearly demonstrate that antagonists have a potential role in the treatment against morphine drug addiction.

\section{Acknowledgements}

This study was supported by the NCRR/RCMI G12 RR03020, the NIGMS/MBRS/SCORE GM08111, and the HRSA SD34HP0 4018 grants.

\section{References}

1. World Health Organization: Cancer Pain Relief. Geneva, 1986.

2. Collin E and Cesselin F: Neurobiological mechanisms of opioid tolerance and dependence. Clin Neuropharmacol 14: 465-488, 1991. 
3. Zhu Z, Badisa RB, Palm DE, et al: Regulation of rat MOR-1 gene expression after chronic ICV administration of morphine. Mol Med Rep 5: 513-516, 2012.

4. Oliveira MT, Rego AC, Morgadinho MT, et al: Macedo: Toxic effects of opioid and stimulant drugs on undifferentiated PC12 cells. Ann NY Acad Sci USA 965: 487-496, 2002.

5. Loh HH and Smith AP: Molecular characterization of opioid receptors. Annu Rev Pharmacol Toxicol 30: 123-147, 1990.

6. Kreek MJ: Opioid receptors: some perspectives from early studies of their role in normal physiology, stress responsivity, and in specific addictive diseases. Neurochem Res 21: 1469-1488, 1996.

7. Borgland SL: Acute opioid receptor desensitization and tolerance: is there a link? Clin Exp Pharmacol Physiol 28: 147-154, 2001.

8. Castelli MP, Melis M, Mameli M, et al: Chronic morphine and naltrexone fail to modify mu-opioid receptor mRNA levels in the rat brain. Brain Res Mol Brain Res 45: 149-153, 1997.

9. Cheung Y-T, Lau WK-W, Yu M-S, et al: Effects of all-transretinoic acid on human SH-SY5Y neuroblastoma as in vitro model in neurotoxicity research. Neurotoxicology 30: 127-135, 2009.

10. Zadina JE, Chang SL, Ge LJ, et al: Mu opiate receptor downregulation by morphine and up-regulation by naloxone in SH-SY5Y human neuroblastoma cells. J Pharmacol Exp Ther 265: 254-262, 1993.

11. Yu VC, Hochhaus G, Chang FG, et al: Differentiation of human neuroblastoma cells: Marked potentiation of prostaglandin E-stimulated accumulation of cyclic AMP by retinoic acid. J Neurochem 51: 1892-1899, 1988.

12. Pahlman S, Ruusala AI, Abrahamsson L, et al: Retinoic acidinduced differentiation of cultured human neuroblastoma cells: a comparison with phorbolester-induced differentiation. Cell Differ 14: 135-144, 1984.

13. Levites Y, Youdim MB, Maor G, et al: Attenuation of 6-hydroxydopamine (6-OHDA)-induced nuclear factor-kappaB (NF-kappaB) activation and cell death by tea extracts in neuronal cultures. Biochem Pharmacol 63: 21-29, 2002.
14. Levites Y, Amit T, Mandel S, et al: Neuroprotection and neurorescue against Abeta toxicity and PKC-dependent release of nonamyloidogenic soluble precursor protein by green tea polyphenol (-)-epigallocatechin-3-gallate. FASEB J 17: 952-954, 2003.

15. Xue S, Jia L and Jia J: Hypoxia and reoxygenation increased BACE1 mRNA and protein levels in human neuroblastoma SH-SY5Y cells. Neurosci Lett 405: 231-235, 2006.

16. Lee JH, Shin SY, Kim S, et al: Suppression of PTEN expression during aggregation with retinoic acid in P19 mouse embryonal carcinoma cells. Biochem Biophys Res Commun 347: 715-722, 2006.

17. Yu X, Mao X, Blake AD, et al: Morphine and endomorphins differentially regulate $\mu$-opioid receptor mRNA in SH-SY5Y human neuroblastoma cells. J Pharmacol Exp Ther 306: 447-454, 2003.

18. Vesanen M, Salminen M, Wessman M, et al: Morphological differentiation of human SH-SY5Y neuroblastoma cells inhibits human immunodeficiency virus type 1 infection. J Gen Virol 75: 201-206, 1994

19. Clagett-Dame M, McNeill EM and Muley PD: Role of all-trans retinoic acid in neurite outgrowth and axonal elongation. $J$ Neurobiol 66: 739-756, 2006.

20. Yabaluri N and Medzihradsky F: Down-regulation of mu-opioid receptor by full but not partial agonists is independent of $G$ protein coupling. Mol Pharmacol 52: 896-902, 1997.

21. Tao PL, Han KF, Wang SD, et al: Immunohistochemical evidence of down-regulation of mu-opioid receptor after chronic PL-017 in rats. Eur J Pharmacol 344: 137-142, 1998.

22. Yamamoto J, Kawamata T, Niiyama Y, et al: Downregulation of $\mathrm{mu}$ opioid receptor expression within distinct subpopulations of dorsal root ganglion neurons in a murine model of bone cancer pain. Neuroscience 151: 843-853, 2008.

23. Xu H, Wang X, Zimmerman D, et al: Chronic morphine up-regulates $\mathrm{G} \alpha 12$ and cytoskeletal proteins in Chinese hamster ovary cells expressing the cloned $\mu$-opioid receptor. J Pharmacol Exp Ther 315: 248-255, 2005. 